\title{
Performance Evaluation of Baffled and Floating Hybrid Constructed Wetland Operated Under Field Conditions
}

\author{
Shrutee Dasare ${ }^{1}$, and Guru Munavalli ${ }^{*}$ \\ ${ }^{1}$ Department of Civil Engineering, Walchand College of Engineering, Sangli, Maharashtra, India
}

\begin{abstract}
Baffled and Floating Hybrid Constructed Wetland (BFHCW) consisting of constructed wetland with brickbat medium and Floating Treatment Wetland (FTW) was developed and operated under actual field flow conditions. BFHCW was vegetated with dual-species (Typha angustifolia L. and Canna indica). The performance of continuously operated system was evaluated for varied Hydraulic Loading Rate (HLR) and Organic Loading Rate (OLR) for a longer period. The system was also assessed for controlled flow rate resulting more uniform OLR and HLR. The results showed that COD removal efficiency was enhanced by 5 to $15 \%$ with controlled flow than uncontrolled highly fluctuating flow conditions. HLR has more impact on COD removal than OLR. BFHCW is effective to an extent of 25 to $40 \%$ for COD removal. BFHCW is found to be cost effective and reasonably efficient treatment for pretreated domestic wastewater.
\end{abstract}

\section{Introduction}

The volume of domestic wastewater generated has increased with population, urbanization, improved living conditions, and economic development. There are two systems for the treatment of domestic wastewater viz. centralized and decentralized. It is disadvantageous to implement centralized systems in developing countries due to financial constraints, requirement of technical expertise for operation and management, and necessity of large sewerage network. Further, wastewater generated from satellite areas or city outskirts require spending even more cost to transfer wastewater to centralized treatment facilities due to the absence of sewer systems. Decentralized Wastewater Treatment System (DWTS) means to treat, reuse or dispose of wastewater at or near its source of generation. DWTS is a cost effective and an appropriate option for small clusters/ household/ housing colony/ institution/ commercial complex. Constructed Wetland (CW) is one of the suitable and affordable options as secondary treatment in DWTS. CWs have emerged as an economical and feasible option for the treatment of domestic wastewater, as they can be built with locally available materials, simple construction, and utilization of natural processes, lower operation and maintenance cost. The aspects related to environmental sustainability and decentralization are present in these treatment technologies [2]. Constructed wetlands (CWs) are proven to be efficient for treating urban and domestic wastewater [1]. The problems associated with $\mathrm{CW}$ such as longer start-up time and high risk of clogging of media resulting waterlogging can hamper the treatment process. The choice of an appropriate coarser media and combining $\mathrm{CW}$ with another treatment system helps to overcome these problems. In this context, CW system with brickbat as a support media for $\mathrm{CW}$ vegetation and Floating Treatment Wetland (FTW) arranged in a typical hybrid sequence can be devised. [4] reported that FTW seem to be effective for the treatment for wastewater and reviewed applicability and design of FTW. A system based on this concept referred as Baffled Floating Hybrid Constructed Wetland (BFHCW) was implemented for the treatment of domestic wastewater in the campus of Walchand College of Engg., Sangli (WCE). BFHCW was a secondary treatment in DWTS of WCE which was fed with wastewater pre-treated in anaerobic baffled reactor.

In the present study, BFHCW was assessed for its performance to remove organic content when operated with actual flow conditions. The primary objective of the work was to study the system behavior under varied Hydraulic Loading Rate (HLR) and Organic Loading Rate (OLR).

\section{Materials and methods}

\subsection{Source of wastewater}

Wastewater is generated from different eating establishments and hostels in WCE campus. It is comprised of washings, septic tank effluent and greywater. The wastewater is pre-treated with bar rack

* Corresponding author: guru.munvalli@walchandsangli.ac.in 
screen and Anaerobic Baffled Reactor (ABR) as part of DWTS. This anaerobically treated wastewater was fed to BFHCW.

\subsection{BFHCW details and operation}

DWTS in WCE is an integrated multi-stage natural wastewater treatment system treating 45 KLD. Wastewater is treated in preliminary and primary treatment consisting of screen chamber and ABR respectively. The effluent from ABR enters BFHCW for secondary treatment. Fig. 1 (a) and (b) shows a schematic sketch and photographic view of BFHCW. BFHCW is made up of 24 compartments with an inlet chamber, alternate compartments having $\mathrm{CW}$ with brickbat as supporting medium [referred as Brickbat Subsurface Wetland (BSFW)], FTW, adsorption bed and an outlet chamber. The effective wastewater depth in the system was $0.80 \mathrm{~m}$. Each BSFW was made up of brickbats of size 50 to $75 \mathrm{~mm}$ with bed porosity of 50 to $60 \%$ and vegetated with dual-species. In FTW different floating mats were tried and that included Thermocol sheet, plastic bottles, and perforated basket. These are shown in Fig. 2 (a) and (b). Charcoal and aggregate were used to provided proper anchorage to vegetation. Dualspecies (Canna indica and Typha angustifolia L). were used in both BSFW and FTW. These were chosen as they were locally available and found to be effective to remove pollutants such as carbonaceous organic matter, nitrogen and phosphorous. Initially, plants were acclimatized by growing them gradually increasing strength of wastewater and then vegetated in BFHCW. An adsorption bed made up of coarse aggregates and charcoal forms the final compartment before outlet chamber. The primary treated wastewater enters BFHCW and flows in an upflow and downflow mode through all the compartments and treated effluent is then discharged into the outlet chamber.

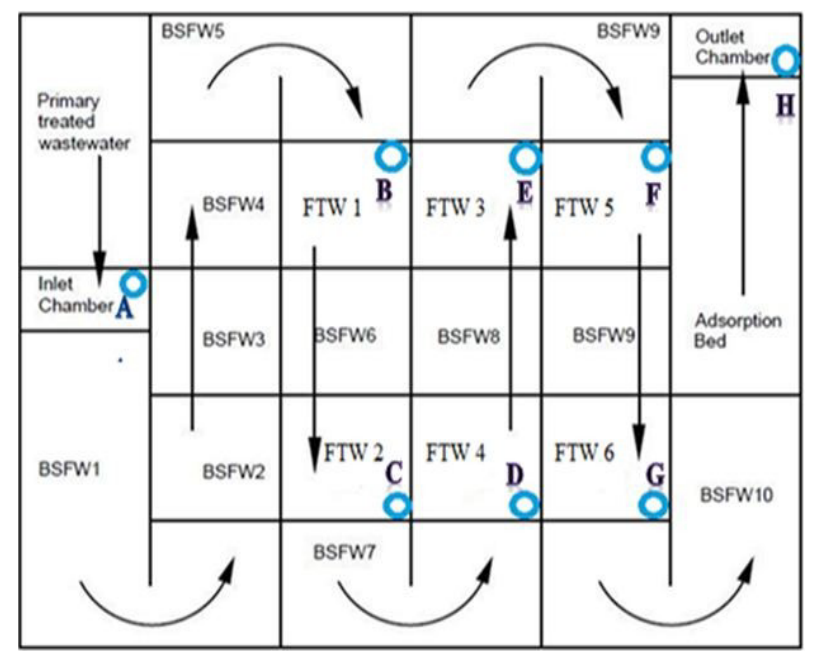

Fig. 1 (a) Schematic line plan of BFHCW

\subsection{Sampling strategy and analysis}

Composite sampling method was adopted. The sampling locations are shown in Error! Reference source not found.(labeled in blue colored circle). Normally samples were collected after every alternate day. Timings for sampling were decided by considering peak flow (9 to $10.30 \mathrm{am}$ ), average flow (3 to $4 \mathrm{pm}$ ) and minimum flow (before $6.20 \mathrm{am}$ ). The parameters such as $\mathrm{pH}, \mathrm{EC}, \mathrm{COD}$, and $\mathrm{BOD}_{3}$ were analyzed to evaluate performance evaluation of the system. The analysis was carried out by referring to APHA [2].

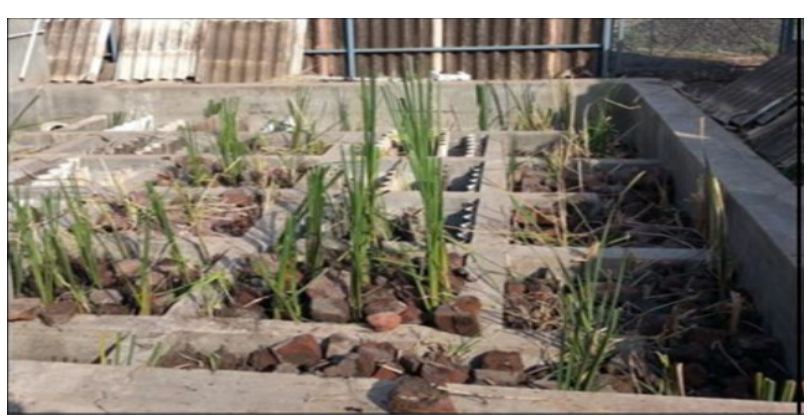

Fig. 2 (b) Photographic view of BFHCW

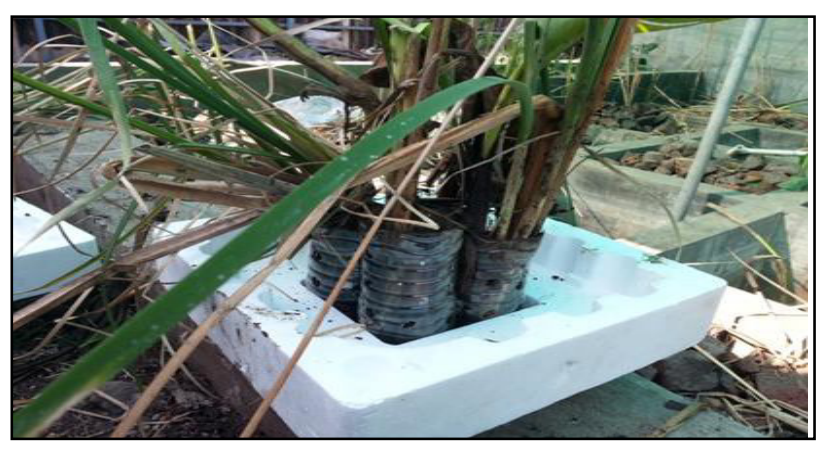

Fig. 2 (a) Photographic view showing configuration of FTW with bio-rack

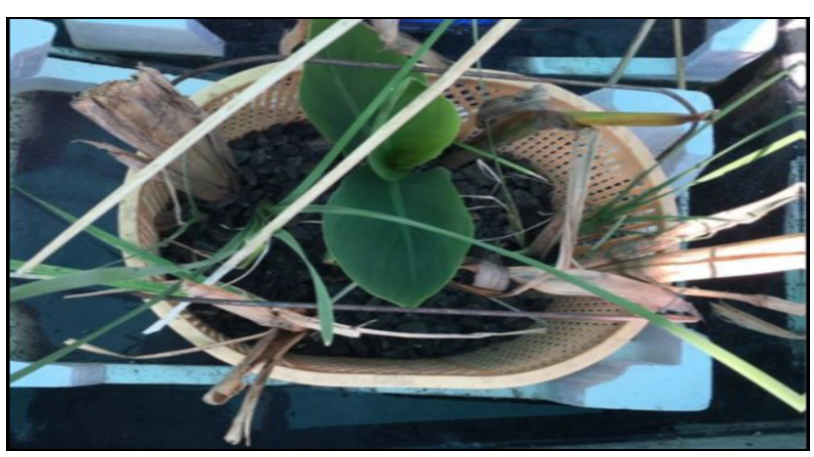

Fig. 2 (b) Photographic view showing configuration of FTW with basket configuration 


\section{Results and Discussions}

\subsection{Influent Characteristics}

Pre-treated wastewater from ABR was taken as an influent to BFHCW system. The summary of influent wastewater characteristics during the study period is given in Error! Reference source not found. $\mathrm{pH}$ values show that wastewater is slightly alkaline in nature. The ratio of $\mathrm{BOD}_{3}$ to $\mathrm{COD}$ is greater than 0.5 indicating its amenability for biological treatment. Thus the ABR effluent is an appropriate feed to BFHCW.

Table 1. Characteristics of influent to BFHCW

\begin{tabular}{|c|c|c|c|}
\hline $\mathrm{pH}$ & $\mathrm{COD}$ & $\mathrm{BOD}_{3} @ 27^{\circ} \mathrm{C}$ & $\mathrm{TKN}$ \\
\hline- & $\mathrm{mg} / \mathrm{L}$ & $\mathrm{mg} / \mathrm{L}$ & $\mathrm{mg} / \mathrm{L}$ \\
\hline $7.35 \pm$ & $268.90 \pm 45.77$ & $144 \pm 7.65$ & 45.54 \\
0.17 & & & \pm 10.43 \\
\hline
\end{tabular}

\subsection{Effect of varied HLR on COD removal}

Error! Reference source not found. shows the effect of varied HLR on COD removal under uncontrolled flow conditions. The results show that the COD removal varies from minimum of $6.2 \%$ to maximum of $47.72 \%$. The corresponding HLR for these removals are 4.8 $\mathrm{m}^{3} / \mathrm{m}^{2} \mathrm{~d}$ and $1.98 \mathrm{~m}^{3} / \mathrm{m}^{2} \mathrm{~d}$ respectively. The COD removal varies inversely with HLR. The observed high and low HLR correspond flow rate of $4000 \mathrm{~L} / \mathrm{h}$ (peak flow) and 1980 L/h (low flow). Hydraulic Retention Time (HRT) was also determined using tracer (Fluoride as tracer) study and found to be 49 min whereas theoretical HRT was $1.30 \mathrm{~h}$ during peak flow. The respective low flow HRT values were $1.66 \mathrm{~h}$ and $2.60 \mathrm{~h}$. Normally, HRT of FTW systems were considerably larger $(>12 \mathrm{~h})$. In the present BHFCW system, HRT values significantly lower than those normally used. The system performance is said to be significantly better owing to the conditions to which it is subjected.

\subsection{Effect of varied OLR on COD removal}

Error! Reference source not found. shows the effect of varied OLR on COD removal. The system was subjected to OLR variation from 0.57 to $1.2 \mathrm{~kg} \mathrm{COD} / \mathrm{m}^{2}$.d. The variation is induced by varying flow rate and influent COD. The COD removal was observed to be in the range 6.2 to $34.18 \%$. The effect of OLR is similar to that of HLR as discussed in the previous section. The COD removal efficiency is not very high but significant considering the high variability of influent COD, lesser HRT, and fluctuating flow rates.

\subsection{Performance evaluation of system based on controlled HLR conditions}

Fig. 6 shows the effect of controlled HLR on COD removal. The trend of COD removal with HLR variation is same as that of uncontrolled conditions. The comparison of the performance of two studies viz. controlled and uncontrolled conditions was done. The system was operated at HLR of $1.71,3.43$ and 4.29 $\mathrm{m}^{3} / \mathrm{m}^{2} . \mathrm{d}$ and OLRs considered were $0.36,0.74$ and 1.44 $\mathrm{m}^{3} / \mathrm{m}^{2} / \mathrm{d}$. Error! Reference source not found. 7 (a) and (b) indicate system performance for COD removal under controlled and uncontrolled conditions for the above specified HLR and OLR values. COD removal rate was observed to be higher by 5 to $15 \%$ for controlled HLR condition than that of uncontrolled condition. Flow normalization and avoidance of sudden change in organic loads are the reasons for relatively better performance under controlled conditions. The results also indicate that HLR is more governing factor than OLR.

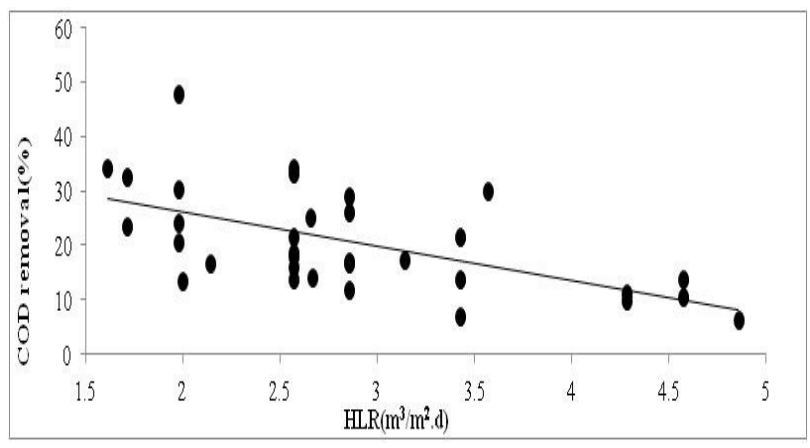

Fig. 3 Effect of varied HLR on COD removal

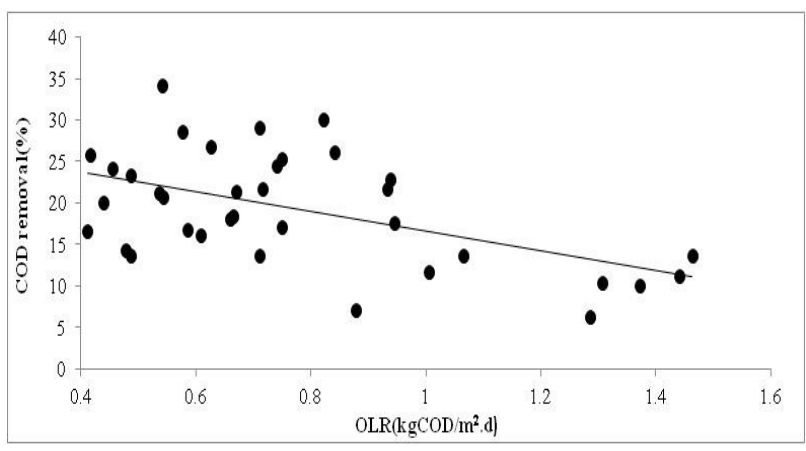

Fig. 4 Effect of varied OLR on COD removal

\subsection{Vegetation development}

The growth of vegetation was monitored during the study in terms of height of plant and root depth. The average initial and final root depth and height of plant are recorded. Plants grew well in BSFW compartments in terms of root depth and plant height. It was observed the pores in the brick-bats media facilitated spaces for deeper root penetration. Vegetation in FTW showed relatively lesser root growth because of no provision of media to support the root development. Compared to Canna indica, Typha angustifolia L. had shown high growth rate due to its dense and fibrous root matrix providing more surface area for attachment. 


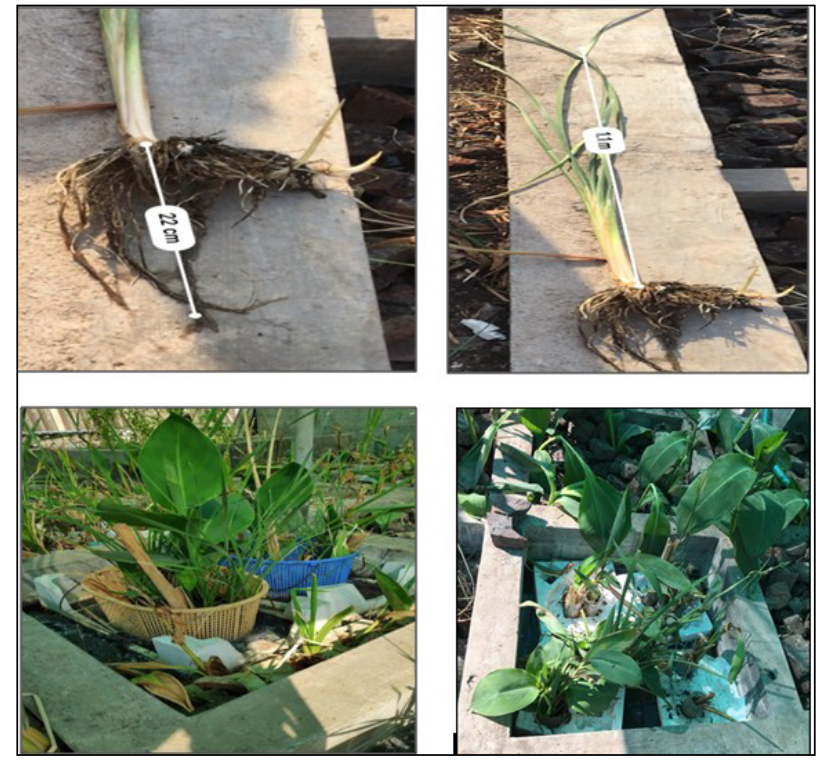

Fig. 5 Photographic view of vegetation with root and height measurement and development of vegetation in FTW at the end of study

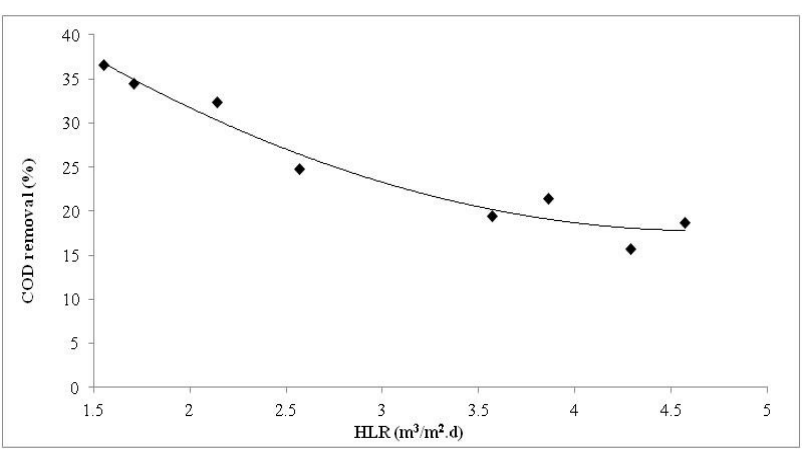

Fig. 6 Effect of controlled HLR on COD removal

\section{Conclusions}

The study was conducted on BFHCW for treatment of an anaerobically pretreated domestic wastewater. The system was operated under high diurnal variation of HLR and OLR. The performance was also assessed when operated under controlled flow conditions leading to uniform HLR and OLR. COD removal rate is increased by 5 to $15 \%$ under controlled conditions. HLR is a dominating parameter compared to OLR and COD removal is significantly affected by varying HLR. BFHCW supported dual-species vegetation. BFHCW is found to be effective for COD removal to an extent of 25 to $40 \%$.

The present study is a part of research project sanctioned by Department of Science and Technology DST) under Water Treatment Initiative (WTI) programme. The authors sincerely acknowledge and thank DST for providing financial support to undertake this study.
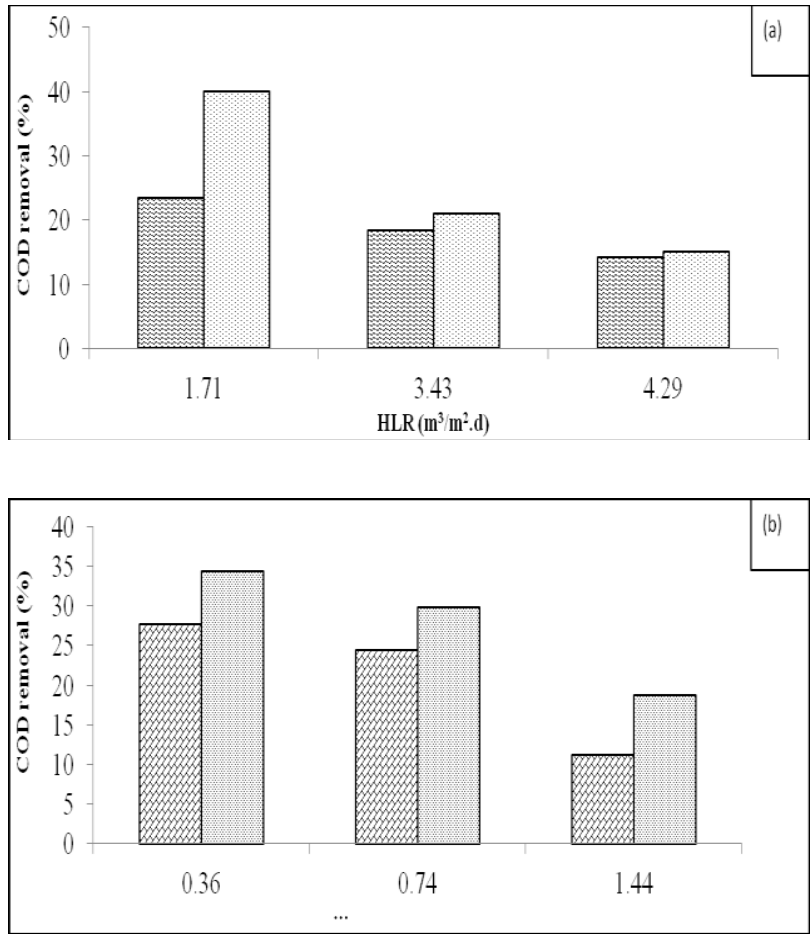

Fig. 7 Comparison of COD removal under controlled and uncontrolled conditions for a) varied HLR (b) varied OLR

\section{References}

1. J. Álvarez, C. Ávila, P. Otter,, R. Kilian, D. Istenič, M. Rolletschek, P. Molle, N. Khalil, I. Ameršek, Mishra, V. K., Jorgensen, C., Garfi, A., P. Carvalho, H. Brix and C. Arias, Water Sci \& Tech. 76 (6), 1474-1489 (2017)

2. APHA, Standard Methods for the Examination of Water and Wastewater, American Water Works Association, Water Environment Federation (2012)

3. G. Dotro, G. Langergraber, P. Molle, J. Nivala, J. Puigagut, O. Stein and M. Von Sperling, IWA Publishing, London, UK, 7, (2017)

4. N. Pavlineri, N. Skoulikidis, V. Tsihrintzis, Chem Engg J. 308, 1120-1132 (2017) 